\title{
Wave Packet Dynamics in a Monolayer Graphene
}

\author{
G. M. Maksimova, V. Ya. Demikhovskii, and E. V. Frolova \\ Nizhny Novgorod State University \\ Russian Federation
}

\section{Introduction}

In the last years the dynamics of wave packets in 2D electron gas and other systems in solids including the phenomenon of Zitterbewegung (ZB) or trembling motion has been the subject of numerous studies. (Shmueli et al.,1995; Schliemann et al.,2005; Schliemann et al.,2006; Schliemann,2008; Zawadzki,2005; Ferrari \& Russo,1990; Zawadzki,2006; Katsnelson,2006; Cserti \& David,2006; Winkler et al.,2007; Trauzettel et al.,2007; Rusin \& Zawadzki,2007; Rusin \& Zawadzki,2007; Rusin \& Zawadzki,2008) Firstly the oscillatory motion analogous to the relativistic Zitterbewegung in two-dimensional systems with the structural and bulk inversion asymmetry was investigated by Schliemann et al.(Schliemann et al.,2005; Schliemann et al.,2006) In the recent work by authors the detailed studying of the electron wave packet dynamics in the semiconductor quantum well under the influence of the Rashba spin-orbit coupling was performed. (Demikhovskii et al.,2008) It was shown (analytically and numerically) that the initial wave packet splits into two parts with different spin polarizations propagating with unequal group velocities. It was demonstrated also that the splitting and overlapping of wave packets leads to the damping of Zitterbewegung.

As well known, the electron Zitterbewegung in relativistic physics at first time was predicted by Schrödinger (Schrödinger,1930) (see also (Barut \& Bracken,1981)). This phenomenon is caused by the interference between positive and negative energy states in the wave packet. The frequency of $\mathrm{ZB}$ motion is determined by the gap between these two states and the amplitude of oscillations in a particle position is of the order of the Compton wave length. This phenomenon was discussed also in Refs.(Lock,1979; Thaller,2004; Braun et al.,1999).

The results of the first experimental observation of $\mathrm{ZB}$ phenomena were published recently in the paper by Gerritsma et. al.(Gerritsma et al.,2010) For the ZB simulation the experimentalists used a linear Paul trap where ion motion can be described by one-dimensional Dirac equation.(Lamata et al.,2007) The authors of Ref. (Gerritsma et al.,2010) study the motion of $\mathrm{Ca}^{+}$ion and determined its position as a function of time for different initial conditions. As was shown in Ref. (Lamata et al.,2007) the solution of the $3+1$ Dirac equation can also be simulated using a single trapped ion with four ionic internal states. In this case the ion position and momentum are associated with respective characteristics of 3D Dirac particle.

In the papers by Rusin and Zawadzki (Rusin \& Zawadzki,2007; Rusin \& Zawadzki,2008) the evolution of the wave packet in a monolayer and bilayer graphene as well as in carbon nanotubes was analyzed. The exact analytical expressions for two components of wave function and average value of position operator were found for bilayer graphene, which allowed to obtain analytical results for the ZB of Gaussian wave packet. It was shown that 
the transient character $\mathrm{ZB}$ in bilayer graphene is due to the fact that wave subpackets related to positive and negative electron energies move in opposite directions, so their overlap diminishes with time. At the same time the dynamics of the wave packets in a monolayer graphene in Ref. (Rusin \& Zawadzki,2007) was not investigated fully.

In this chapter we present the detailed description of wave packet evolution including the phenomenon of $\mathrm{ZB}$ of the packet center in a monolayer graphene (see also Ref. (Maksimova et al.,2008)). The analytical expressions for the components of wave function at $t>0$ are found in the form of two-dimensional integrals. Using these equations we obtain the full electron probability density and such dynamical characteristics of the packet center as the average components of coordinate and velocity. We investigate the influence of the initial pseudospin polarization on the space-time evolution of the wave packet, in particular, on the trajectory of its center. As a result, the direction of the packet motion is determined not only by the orientation of the average momentum, but mainly by the phase difference between the up- and low-components of the wave functions. Our analytically results are illustrated by a graphic presentation. The obtained results can be useful for analysis of the functioning of graphene's electronic structures and devices.

\section{Basic equations}

Graphene is a single layer of carbon atom densely packed in a honeycomb lattice. The two-dimensional Hamiltonian describing its band structure has the form (Wallace,1947; Slonczewski \& Weiss,1958; Novoselov et al.,2005; Novoselov et al.,2006; Zhang et al.,2005)

$$
\hat{H}=u \vec{\sigma} \hat{\vec{p}},
$$

where $u \approx 10^{8} \mathrm{~cm} / \mathrm{s}, \hat{\vec{p}}=\left(\hat{p}_{x}, \hat{p}_{y}\right)$ is the momentum operator defined with respect to the centre of the valley centered at the corner of the Brillouin zone with wave vector $\vec{K}$. Pauli matrices $\sigma_{i}$ operate in the space of the electron amplitude on two sites $(A$ and $B)$ in the unit cell of a hexagonal crystal. This internal degree of freedom plays a role of a pseudospin. The Dirac-like Hamilton $\hat{H}$ determines the linear dispersion relation

$$
E_{p, s}=\sup .
$$

Here $p=\sqrt{p_{x}^{2}+p_{y}^{2}}, s=1$ for the electron in the conduction band and $s=-1$ for the valence band ("hole" branch of quasiparticles). The corresponding eigenfunctions are given by

$$
\varphi_{\vec{p}, s}(\vec{r}, t)=\frac{1}{2 \sqrt{2} \pi \hbar} \exp \left(i \frac{\vec{p} \vec{r}}{\hbar}-i \frac{E_{p, s} t}{\hbar}\right)\left(\begin{array}{c}
1 \\
s \mathrm{e}^{i \varphi}
\end{array}\right),
$$

with $\mathrm{e}^{i \varphi}=\frac{p_{x}+i p_{y}}{p}$.

The time-evolution of an arbitrary initial state $\psi(\vec{r}, 0)$ in Shrödinger representation can be found with the help of Green's function $G_{\mu v}\left(\vec{r}, \overrightarrow{r^{\prime}}\right)$

$$
\psi_{\mu}(\vec{r}, t)=\int G_{\mu v}\left(\vec{r}, \overrightarrow{r^{\prime}}, t\right) \psi_{v}\left(\overrightarrow{r^{\prime}}, 0\right) d \overrightarrow{r^{\prime}},
$$

where $\mu, v=1,2$ are matrix indices, corresponding to the upper and lower components of $\psi(\vec{r}, t)$. These components are related to the probability of finding electron at the sites of the sublattices $A$ and $B$ correspondingly. The standard expression for Green's function is 


$$
G_{\mu v}\left(\vec{r}, \vec{r}^{\prime}, t\right)=\sum_{s= \pm 1} \int d \vec{p} \varphi_{\vec{p}, s ; \mu}(\vec{r}, t) \varphi_{\vec{p}, s ; v}^{*}\left(\vec{r}^{\prime}, 0\right)
$$

Using Eq.(3) for $\varphi_{\vec{p}, s ; \mu}(\vec{r}, t)$ we find

$$
\begin{gathered}
G_{11}\left(\vec{r}, \vec{r}^{\prime}, t\right)=G_{22}\left(\vec{r}, \overrightarrow{r^{\prime}}, t\right)=\frac{1}{(2 \pi \hbar)^{2}} \int \exp \left(i \frac{\vec{p}\left(\vec{r}-\overrightarrow{r^{\prime}}\right)}{\hbar}\right) \cos \left(\frac{u p t}{\hbar}\right) d \vec{p}, \\
G_{21}\left(\vec{r}, \vec{r}^{\prime}, t\right)=G_{12}^{*}\left(\vec{r}, \vec{r}^{\prime}, t\right)=\frac{-i}{(2 \pi \hbar)^{2}} \int \frac{p_{x}+i p_{y}}{p} \exp \left(i \frac{\vec{p}\left(\vec{r}-\vec{r}^{\prime}\right)}{\hbar}\right) \sin \left(\frac{u p t}{\hbar}\right) d \vec{p},
\end{gathered}
$$

Let us represent the initial wave function by Gaussian wave packet having the width $d$ and nonvanishing average momentum $p_{0 y}=\hbar k_{0}$

$$
\begin{aligned}
& \psi(\vec{r}, 0)=\frac{f(\vec{r})}{\sqrt{\left|c_{1}\right|^{2}+\left|c_{2}\right|^{2}}}\left(\begin{array}{l}
c_{1} \\
c_{2}
\end{array}\right), \\
& f(\vec{r})=\frac{1}{d \sqrt{\pi}} \exp \left(-\frac{r^{2}}{2 d^{2}}+i k_{0} y\right),
\end{aligned}
$$

where coefficients $c_{1}$ and $c_{2}$ determine the initial pseudospin polarization. We suppose that the packet width $d$ is much greater than the lattice period and consequently $\psi(\vec{r}, 0)$ is smooth enveloping function. We suppose also that the most of the states in valence band are unfilled, that corresponds to negative Fermi level located far from Dirac point (see also Ref. (Rusin \& Zawadzki,2008)). Substituting Eqs.(8a, 8b) in Eq.(4) and using the expressions (6) and (7) we obtain

$$
\begin{gathered}
\psi_{1}(\vec{r}, t)=\frac{1}{\sqrt{\left|c_{1}\right|^{2}+\left|c_{2}\right|^{2}}}\left(c_{1} \phi_{1}(\vec{r}, t)-c_{2} \phi_{2}(-x, y, t)\right), \\
\psi_{2}(\vec{r}, t)=\frac{1}{\sqrt{\left|c_{1}\right|^{2}+\left|c_{2}\right|^{2}}}\left(c_{2} \phi_{1}(\vec{r}, t)+c_{1} \phi_{2}(\vec{r}, t)\right),
\end{gathered}
$$

where, for notational convenience, $\phi_{1,2}(\vec{r}, t)$ denote the functions

$$
\begin{gathered}
\phi_{1}(\vec{r}, t)=\int G_{11}\left(\vec{r}, \overrightarrow{r^{\prime}}, t\right) f\left(\overrightarrow{r^{\prime}}, 0\right) d \overrightarrow{r^{\prime}}=\frac{d \mathrm{e}^{-\left(k_{0} d\right)^{2} / 2}}{2 \hbar^{2} \sqrt{\pi^{3}}} \times \\
\times \int \exp \left(i \frac{\overrightarrow{p r}}{\hbar}-\frac{p^{2} d^{2}}{2 \hbar^{2}}+\frac{p_{y} k_{0} d^{2}}{\hbar}\right) \cos \left(\frac{u p t}{\hbar}\right) d \vec{p}, \\
\phi_{2}(\vec{r}, t)=\int G_{21}\left(\vec{r}, \overrightarrow{r^{\prime}}, t\right) f\left(\overrightarrow{r^{\prime}}, 0\right) d \overrightarrow{r^{\prime}}=\frac{-i d \mathrm{e}^{-\left(k_{0} d\right)^{2} / 2}}{2 \hbar^{2} \sqrt{\pi^{3}}} \times \\
\times \int \frac{p_{x}+i p_{y}}{p} \exp \left(i \frac{\vec{p} \vec{r}}{\hbar}-\frac{p^{2} d^{2}}{2 \hbar^{2}}+\frac{p_{y} k_{0} d^{2}}{\hbar}\right) \sin \left(\frac{u p t}{\hbar}\right) d \vec{p} .
\end{gathered}
$$

Using the cylindrical coordinates in Eqs.(11), (12) and integrating over the angular variable, we have 


$$
\begin{gathered}
\phi_{1}(\vec{r}, t)=\frac{\mathrm{e}^{-\frac{a^{2}}{2}}}{d \sqrt{\pi}} \int_{0}^{\infty} \mathrm{e}^{-\frac{q^{2}}{2}} \cos (q t) J_{0}\left(q \sqrt{r^{2}-a^{2}-2 i a y}\right) q d q, \\
\phi_{2}(\vec{r}, \tau)=\frac{\mathrm{e}^{-\frac{a^{2}}{2}}}{d \sqrt{\pi}} \frac{x+a+i y}{\sqrt{r^{2}-a^{2}-2 i a y}} \int_{0}^{\infty} \mathrm{e}^{-\frac{q^{2}}{2}} \sin (q t) J_{1}\left(q \sqrt{r^{2}-a^{2}-2 i a y}\right) q d q,
\end{gathered}
$$

where $J_{0}(z), J_{1}(z)$ are Bessel functions. For the sake of convenience we introduce in Eqs.(13), (14) and everywhere below the dimensionless variables, measuring the distance in the units of initial width of wave packet $d$ and time in $d / u$ units. Besides, instead of the wave vector $k_{0}$ we consider the parameter $a=k_{0} d$.

\section{Zitterbewegung of Gaussian wave packet with different pseudospin polarization}

Now we describe the time dynamics of Gaussian wave packets, in particular, the ZB phenomenon and the influence of the initial pseudospin polarization on the characteristics of trembling motion.

i). Following Ref. (Rusin \& Zawadzki,2007) let us firstly consider the model problem when the lower component of initial wave function is equal to zero, i.e. the parameters $c_{1}=1, c_{2}=0$ in Eq.(8a). That means that at the initial moment of time the electron probability is located at the sites of the sublattice $A$. It is not difficult to show that this packet is formed by the states with positive and negative energies. The relative weight of these states is equal to one. The wave function for $t>0$ can be found using Eqs.(9), (10):

$$
\psi(\vec{r}, t)=\left(\begin{array}{c}
\phi_{1}(\vec{r}, t) \\
\phi_{2}(\vec{r}, t)
\end{array}\right)
$$

where the functions $\phi_{1}(\vec{r}, t), \phi_{2}(\vec{r}, t)$ are defined by Eqs.(13),(14).

In Fig.1 we represent the full electron density at the moment $t=7$ for initial wave packet, Eq.(8b) with width $d=2 \mathrm{~nm}$ and $k_{0}=0.6 \mathrm{~nm}^{-1}$. As one can see, at $t>0$ this packet splits in two parts moving along $y$ axis with opposite velocities so that the electron probability density is symmetrical with respect to $y: \rho(x, y, t)=\rho(x,-y, t)$. Note that at the case $k_{0}=0$ the electron probability density has a cylindrical symmetry at all time. Indeed it is easy to see that the initial wave function is the eigenstate of total "angular momentum" operator $\hat{I}_{z}=\hat{l}_{z}+\hbar / 2 \sigma_{z}\left(\hat{l}_{z}=-i \hbar \partial / \partial \alpha\right) \hat{I}_{z} \Psi(\vec{r}, 0)=\hbar / 2 \Psi(\vec{r}, 0)$. Since $I_{z}$ is a conserved quantity the wave function $\Psi(\vec{r}, t)=\left(\Psi_{1}(\vec{r}, t), \Psi_{2}(\vec{r}, t)\right)^{T}$ obeys this equation too. It follows that only lower component depends on $\alpha: \Psi_{2}(\vec{r}, t) \sim e^{i \alpha} f_{2}(\varrho, t)$ (see Eqs.(13)-15) for $\left.k_{0}=0\right)$. Thus the probability density remains an axially symmetric at $t>0$. For enough large time the width of both parts of the packet with $k_{0} \neq 0$ increases with time due to effect of dispersion. One can check that in this situation the contributions of two components of wave functions $\psi_{1}(\vec{r}, t)$ and $\psi_{2}(\vec{r}, t)$ in full electron density are equal. In other words the electron probability distributes with the time on the sides of sublattice $A$ and $B$. Note at the same time $\rho(x, y, t) \neq \rho(-x, y, t)$ and the packet center oscillates along $x$ direction (Zitterbewegung).

To analyze this motion we find the average value of position operator. To do it, we use the momentum representation. The upper $\left(C_{1}(\vec{p}, t)\right)$ and lower $\left(C_{2}(\vec{p}, t)\right)$ components of wave function (15) in this representation can be easily obtained from Eqs.(11), (12). After that the usual definition 


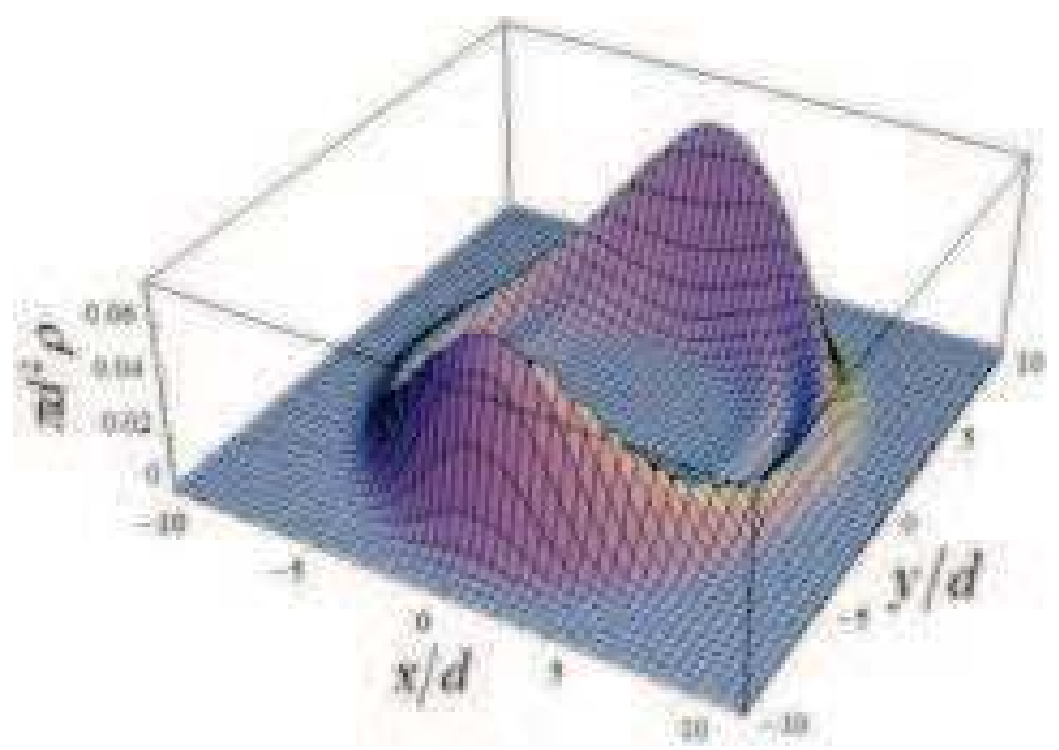

Fig. 1. (Color on line). The electron probability density $\rho(\vec{r}, t)=\left|\psi_{1}\right|^{2}+\left|\psi_{2}\right|^{2}$ for initial wave packet determined by Eqs.(8a), (8b) with $c_{1}=1$ and $c_{2}=0$ for $a=k_{0} d=1.2$ at the time $t=7$ (in the units of $d / u$ ).

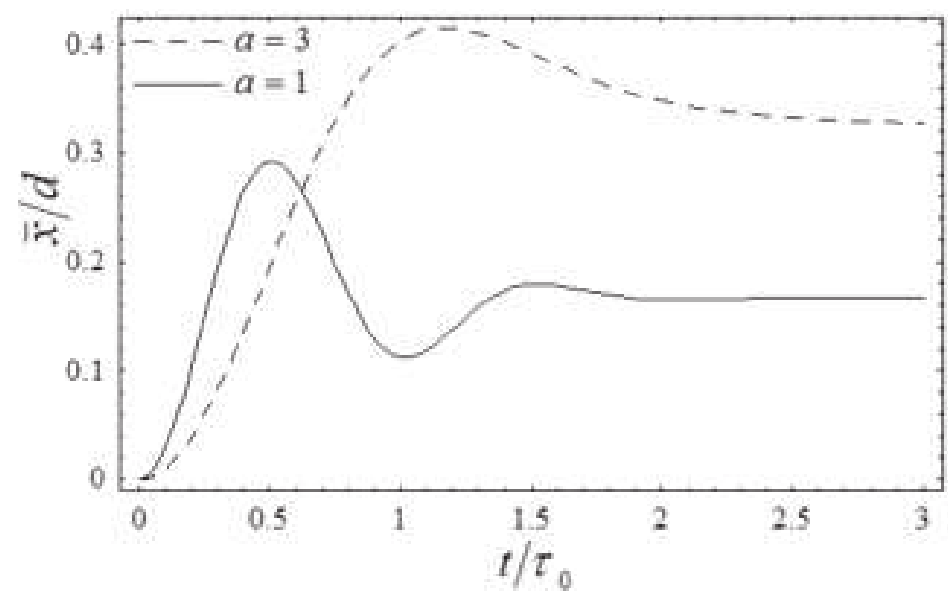

Fig. 2 . The average coordinate $\bar{x}(t)$ versus time $\left(\tau_{0}=d / u\right)$ for the wave packet with initial pseudospin polarization along $z$ axis for two values of $a$.

$$
\overline{\vec{r}}(t)=\sum_{j=1}^{2} \int d \vec{p} C_{j}^{*}(\vec{p}, t) i \hbar \frac{d C_{j}(\vec{p}, t)}{d \vec{p}},
$$

readily yields 


$$
\begin{gathered}
\bar{y}(t)=0, \\
\bar{x}(t)=\frac{1-\mathrm{e}^{-a^{2}}}{2 a}-\mathrm{e}^{-a^{2}} \int_{0}^{\infty} \mathrm{e}^{-q^{2}} \cos (2 q t) I_{1}(2 a q) d q,
\end{gathered}
$$

where $I_{1}(z)$ is a modified Bessel function of the first order. In Eq.(17b) the integral term represents the Zitterbewegung. Note that average value $\bar{x}(t)$ depends on only one parameter $a$ (in the dimensionless variables). The obtained functions $\bar{x}(t)$ which describes the typical transient Zitterbewegung are plotted in Fig.2. After the oscillation disappears the center of the packet is displaced by amount which equals to the first term Eq.(15). In the case when the wave packet width is large enough and the inequality $a=d k_{0} \gg 1$ takes place, Eq.(17b) reduces to

$$
\bar{x}(t)=\frac{1-\mathrm{e}^{-t^{2}} \cos (2 a t)}{2 a} .
$$

The details of the calculation of asymptotic formula similar to Eq. (18) are represented in Ref. (Demikhovskii et al.,2008).

As it follows from Eqs.(17), (18) for given initial polarization of wave packet the ZB occurs in the direction perpendicular to the initial momentum $p_{0 y}=\hbar k_{0}$, just as for bilayer graphene (Rusin \& Zawadzki,2007) and for the semiconductor quantum well in the presence of the Rashba spin-orbit coupling (Schliemann et al.,2005; Schliemann et al.,2006; Demikhovskii et al.,2008). One can see from Eq.(18) that the trembling motion has a transient character as it was described in Refs. (Rusin \& Zawadzki,2007; Demikhovskii et al.,2008) and at $t \gg 1 x(t) \rightarrow 1 / 2 a$. We should notice that Eqs.(17b), (18) coincide with corresponding formulas of Ref. (Demikhovskii et al.,2008). This is because the Hamiltonian for the system with Rashba-coupling

$$
H_{R}=\frac{\hat{\vec{p}}^{2}}{2 m}+\alpha\left(\hat{p}_{y} \hat{\sigma}_{x}-\hat{p}_{x} \hat{\sigma}_{y}\right),
$$

where $\alpha$ is a Rashba coupling constant, transforms into Hamiltonian for monolayer graphene, Eq.(1), if we make the replacement in Eq.(19)

$$
x \rightarrow-y^{\prime}, \quad y \rightarrow x^{\prime}, \quad \alpha \rightarrow u, \quad m \rightarrow \infty .
$$

ii). Let us consider now the case when $c_{1}=c_{2}=1$, that is pseudospin is directed along $x$ axis at $t=0$. Then from Eqs.(9), (10)

$$
\psi(\vec{r}, t)=\frac{1}{\sqrt{2}}\left(\begin{array}{c}
\phi_{1}(\vec{r}, t)-\phi_{2}(-x, y, t) \\
\phi_{1}(\vec{r}, t)+\phi_{2}(\vec{r}, t)
\end{array}\right) .
$$

Fig.3 illustrates the corespondent electron probability density at the time moment $t=7$ for initial wave packet, Eq.(8b), for the same parameters as in Fig.1. One can see that the initial wave packet at $t>0$, as in previous case, splits into two parts propagating along $y$ in opposite directions so that the symmetry concerning this axis, i.e. $\rho(x, y, t)=\rho(x,-y, t)$, retain during the time (as the case $\mathrm{i})$ ). The distribution of the probability density along $x$ axis clearly demonstrates that its maximum is displaced in the positive direction that corresponds to the motion of the packet centre along $x$ axis. The velocity of such motion $\bar{v}_{x}=\frac{d \bar{x}}{d t}$ consists 


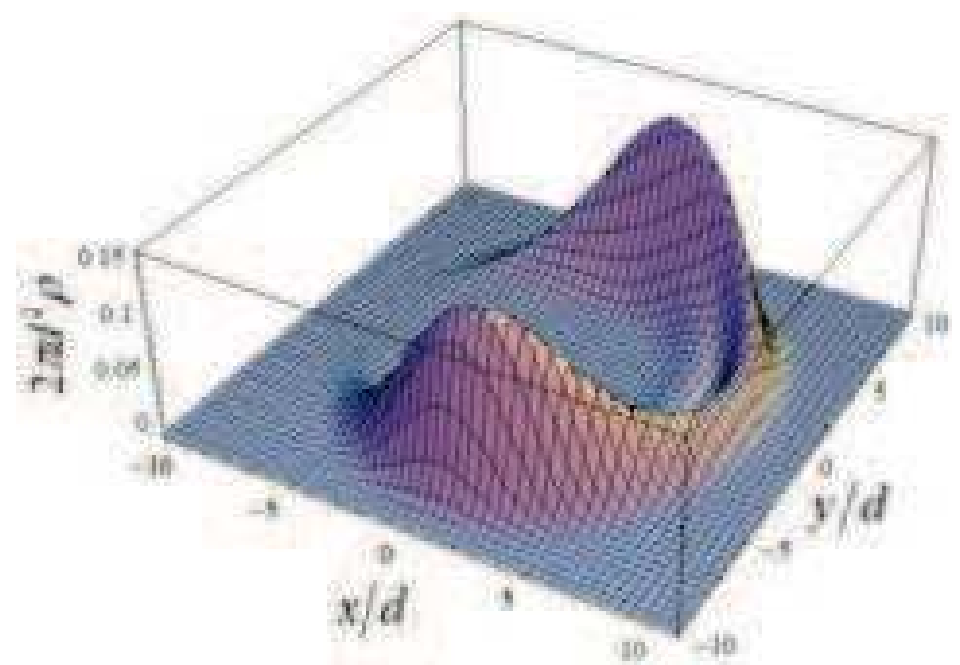

Fig. 3. (Color on line). The electron probability density $\rho(\vec{r}, t)=\left|\psi_{1}\right|^{2}+\left|\psi_{2}\right|^{2}$ for initial wave packet, Eqs.(8a), (8b) with $c_{1}=1$ and $c_{2}=1$ for $a=k_{0} d=1.2$ at the time $t=7$ (in the units of $d / u)$.

of both constant as well as oscillatory parts. Really, a straightforward calculation yields the average value of position operator $x$

$$
\bar{x}(t)=\frac{1-\mathrm{e}^{-a^{2}}}{2 a^{2}} t+\frac{\mathrm{e}^{-a^{2}}}{2 a} \int_{0}^{\infty} \mathrm{e}^{-q^{2}} \sin (2 q t) \frac{d}{d q} I_{1}(2 a q) d q,
$$

and $\bar{y}(t)=0$ like for the case i). In Fig.4 we demonstrate the dependence $\bar{x}(t)$ for various values of parameter $a$. When the parameter $a$ increases, the amplitude of ZB and the period of oscillations decrease. At $a \gg 1$ we have from Eq.(22)

$$
\bar{x}(t)=\frac{t}{2 a^{2}}+\frac{1}{2 a} \mathrm{e}^{-t^{2}} \sin (2 a t) .
$$

We see that the character of motion of wave packet is changed. Now the center of wave packet moves along $x$ direction with constant velocity, which is determined by the first term in Eqs.(22), (23) and performs the damping oscillations. It is not difficult to find the constant component $\overrightarrow{\vec{V}}_{0}$ (in the units of $u$ ) of wave packet velocity for arbitrary initial wave function (8a) (see Appendix):

$$
\begin{aligned}
& \bar{V}_{0 x}=\frac{2}{\left|c_{1}\right|^{2}+\left|c_{2}\right|^{2}} \int d \vec{p}|F(\vec{p})|^{2}\left(\frac{p_{x}^{2}}{p^{2}} \operatorname{Re}\left(c_{1}^{*} c_{2}\right)+\frac{p_{x} p_{y}}{p^{2}} \operatorname{Im}\left(c_{1}^{*} c_{2}\right)\right), \\
& \bar{V}_{0 y}=\frac{2}{\left|c_{1}\right|^{2}+\left|c_{2}\right|^{2}} \int d \vec{p}|F(\vec{p})|^{2}\left(\frac{p_{x} p_{y}}{p^{2}} \operatorname{Re}\left(c_{1}^{*} c_{2}\right)+\frac{p_{y}^{2}}{p^{2}} \operatorname{Im}\left(c_{1}^{*} c_{2}\right)\right) .
\end{aligned}
$$

In particular for $F(\vec{p})=F(-\vec{p})$ and $c_{1}=c_{2}=1$ Eqs.(24),(25) give $\bar{V}_{x 0}=1 / 2, \bar{V}_{y 0}=0$. This result also follows from Eq.(22) when $k_{0}=0$ or for the narrow-width Gaussian wave packet $\left(a=k_{0} d \rightarrow 0\right)$. When the width of packet is increased the velocity of motion of its centre is 


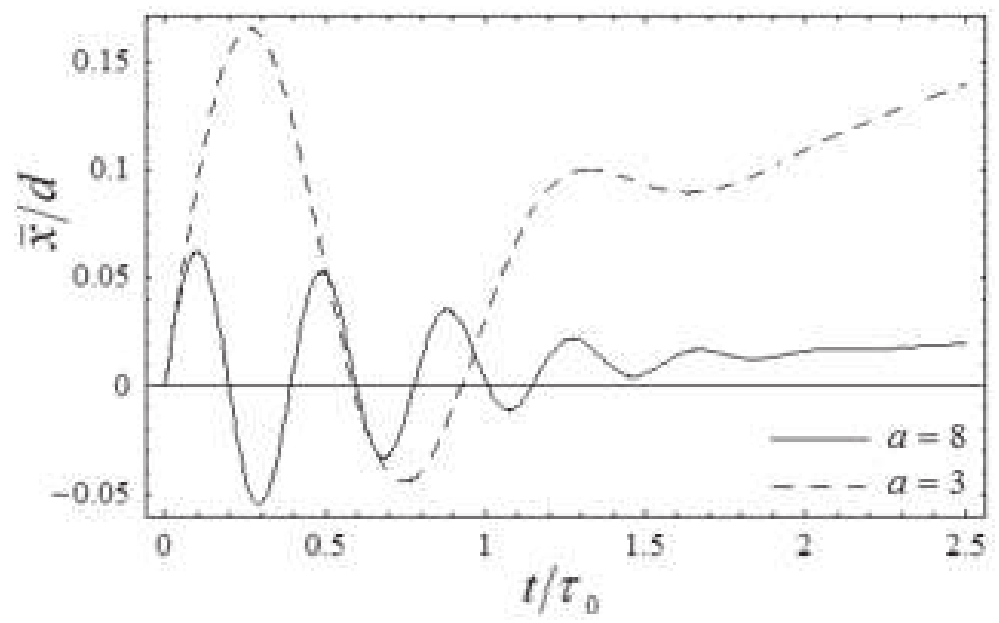

Fig. 4 . The average coordinate $\bar{x}(t)$ versus time $\left(\tau_{0}=d / u\right)$ for the wave packet with initial pseudospin polarization along $x$ axis for different values of $a$.

decreased. The frequency and amplitude of the Zitterbewegung for $a \gg 1$ are the same as in the case i). However, the first term in Eq.(22) corresponding to the motion of wave packet with constant velocity reduces the effect of ZB at least for $a \lesssim 1$ (Fig.4).

It is not difficult to show that as in the other two-band systems the phenomenon of $\mathrm{ZB}$ in graphene is a result of an interference of states corresponding to positive and negative eigenenergies of Hamiltonian, Eq.(1). For wide enough packet $a=k_{0} d \gg 1$ and at time $t>1$ when the ZB disappears two split parts of initial wave packet (see Fig. 3) move along $y$ axis with opposite velocities $u / 2$ and $-u / 2$. In this situation the subpackets moving in the positive and negative directions consist of the states with positive and negative energies correspondingly.

iii). When the initial pseudospin is along $y$ axis the wave function at $t>0$ has the form

$$
\psi(\vec{r}, t)=\frac{1}{\sqrt{2}}\left(\begin{array}{c}
\phi_{1}(\vec{r}, t)-i \phi_{2}(-x, y, t) \\
i \phi_{1}(\vec{r}, t)+\phi_{2}(\vec{r}, t)
\end{array}\right) .
$$

In Fig.5 the full electron density for the same moment of time and for the same parameters as in previous cases is shown. As one can see, the initial wave packet does not split into two parts at $t>0$ unlike in the cases i) and ii). This result is confirmed by the straightforward calculations. Indeed, one can show that the eigenenergy states corresponding to propagation in the positive direction along $y$ axis give the dominant contribution in total wave function, Eq.(26). For wide packets $(a \gg 1)$ almost all of these states belong to the positive branch of energy.

The results of calculations of average values of $x$ and $y$ for this polarization lead to

$$
\begin{gathered}
\bar{x}(t)=0 \\
\bar{y}(t)=\left(1-\frac{1}{2 a^{2}}+\frac{\mathrm{e}^{-a^{2}}}{2 a^{2}}\right) t+\frac{\mathrm{e}^{-a^{2}}}{2 a} \int_{0}^{\infty} \mathrm{e}^{-q^{2}} \sin (2 q t) I_{1}(2 a q) \frac{d q}{q} .
\end{gathered}
$$




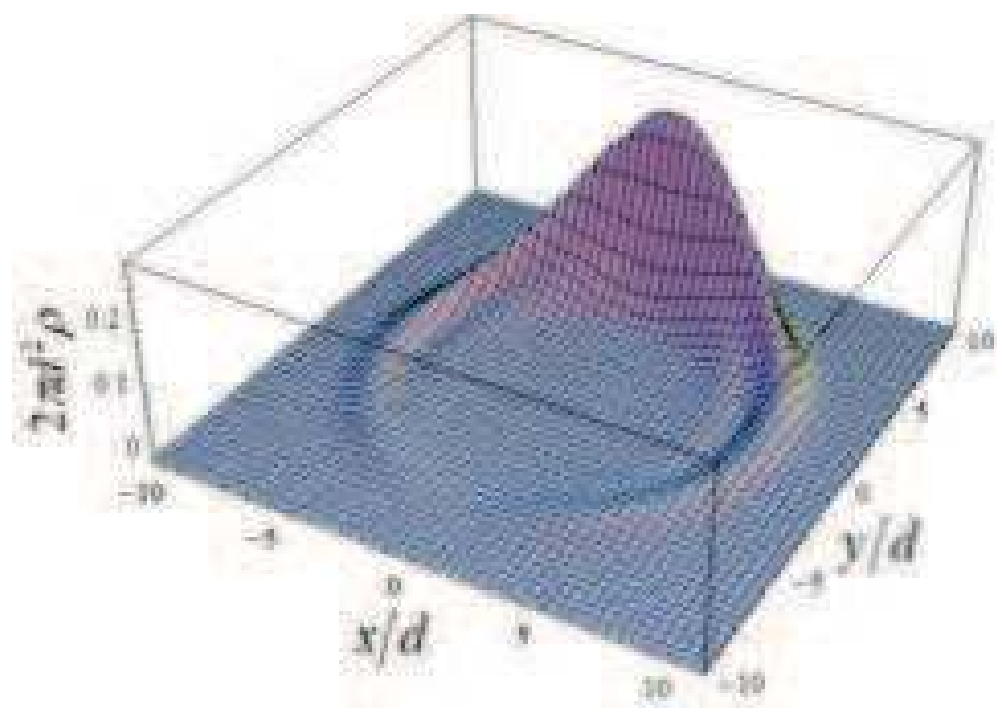

Fig. 5. (Color online). The electron probability density $\rho(\vec{r}, t)=\left|\psi_{1}\right|^{2}+\left|\psi_{2}\right|^{2}$ for initial wave packet, Eqs.(8a), (8b) with $c_{1}=1$ and $c_{2}=i$ for $a=k_{0} d=1.2$ at time $t=7$ (in the units of $d / u)$.

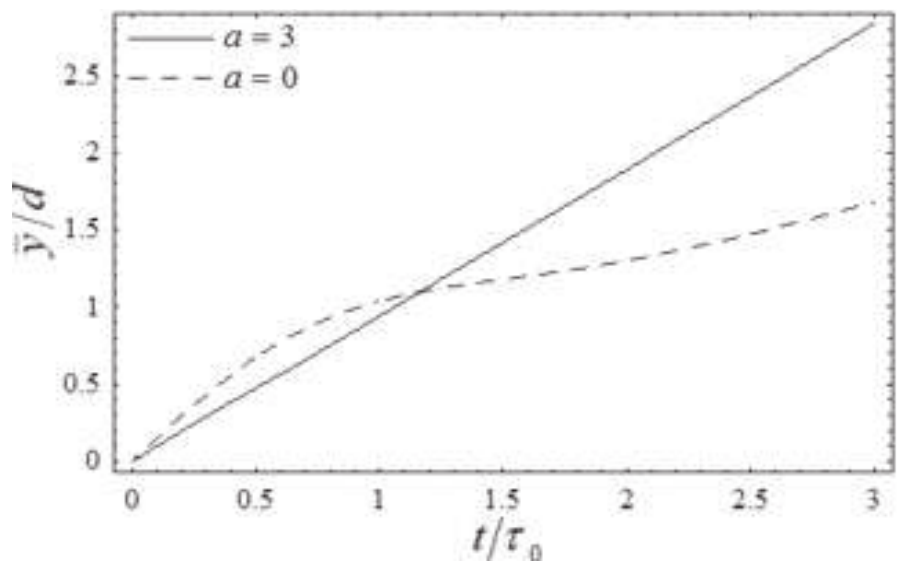

Fig. 6 . The average coordinate $\bar{y}(t)$ versus time $\left(\tau_{0}=d / u\right)$ for the wave packet with initial pseudospin polarization along $y$ axis for different values of $a$.

Thus in the considered case the wave packet propagates along $y$ axis and the Zitterbewegung has the "longitudinal" character. It is interesting to note that $1 D$ wave packet

$$
\psi(y, 0)=F(y)\left(\begin{array}{l}
1 \\
i
\end{array}\right)
$$


where $F(y)$ is arbitrary function, propagates along $y$ direction without changing of its form

$$
\psi(y, t)=F(y-u t)\left(\begin{array}{l}
1 \\
i
\end{array}\right) .
$$

Note that in a numerical work (Thaller,2004) the author has observed similar oscillatory behavior of the expectation value of the position operator for one - dimensional relativistic electron in vacuum. In Ref.(Thaller,2004) it was also shown that apart from the rapid oscillation, the wave packet drifts slowly even when its average momentum is zero.

In Fig. 6 we represent the dependence $\bar{y}(t)$ for different values of parameter $a$. As one can see, even at zero value of $a$ the oscillations are absent. In this case, as it follows from Eqs.(25),(28) the drift velocity is equal to $1 / 2$ (in the units of $u$ ). As above, Eq.(28), takes more simple form at $a \gg 1$

$$
\bar{y}(t)=t+\frac{1}{4 a^{3}} \mathrm{e}^{-t^{2}} \sin (2 a t) .
$$

Comparing Eqs.(18), (23), (29), we see that the amplitude for the "longitudinal" Zitterbewegung is much smaller than the amplitude of "transverse" Zitterbewegung. This fact can bee seen as a consequence of special form of the initial wave function, which in the given case consists of (at $a \gg 1$ ) the states with positive energy mostly. That makes the interference between the positive and negative components difficult, i.e. decreases the ZB. Moreover, at any values of the parameter $a$ the integral term in Eq.(28), corresponding to the oscillating motion, is negligible in comparison with the first term, and one may neglect the effect of the "longitudinal" ZB.

As was demonstrated above, the direction of the average velocity depends not only on module of the components $\psi_{1}(\vec{r}, 0)$ and $\psi_{2}(\vec{r}, 0)$, but also on their phases. Specifically for the initial Gaussian packet

$$
\psi(\vec{r}, 0)=\frac{f(\vec{r})}{\sqrt{2}}\left(\begin{array}{c}
1 \\
\mathrm{e}^{i \varphi}
\end{array}\right),
$$

the probability density becomes asymmetric and the average position operator has two components

$$
\overline{\mathbf{r}}(t)=\bar{x}(t) \cos \varphi \overrightarrow{\mathbf{e}}_{x}+\bar{y}(t) \sin \varphi \overrightarrow{\mathrm{e}}_{y},
$$

where $\varphi$ is an arbitrary phase difference between the up and low components of wave function and $\bar{x}(t), \bar{y}(t)$ are determined by Eqs.(22), (28). For illustration we show in Fig.7 the electron probability density obtained for the initial packet, Eq.(30), with $\varphi=\pi / 4$.

It is clear that the phase $\varphi$ determines the direction of the average velocity of the packet center. Using the expression for velocity operator $\hat{\vec{v}}=u \vec{\sigma}$ and Eq.(30) we obtain (in the dimensionless variables) at $t=0$ :

$$
\bar{v}_{x}(0)=\cos \varphi, \quad \bar{v}_{y}(0)=\sin \varphi .
$$

The components of the velocity for a large enough time, when the trembling motion stops, can be found from Eqs.(22),(28) and (31) for arbitrary parameter $a$

$$
\bar{v}_{x}=\frac{1-\exp \left(-a^{2}\right)}{2 a^{2}} \cos \varphi, \bar{v}_{y}=\left(1-\frac{1}{2 a^{2}}+\frac{\exp \left(-a^{2}\right)}{2 a^{2}}\right) \sin \varphi .
$$




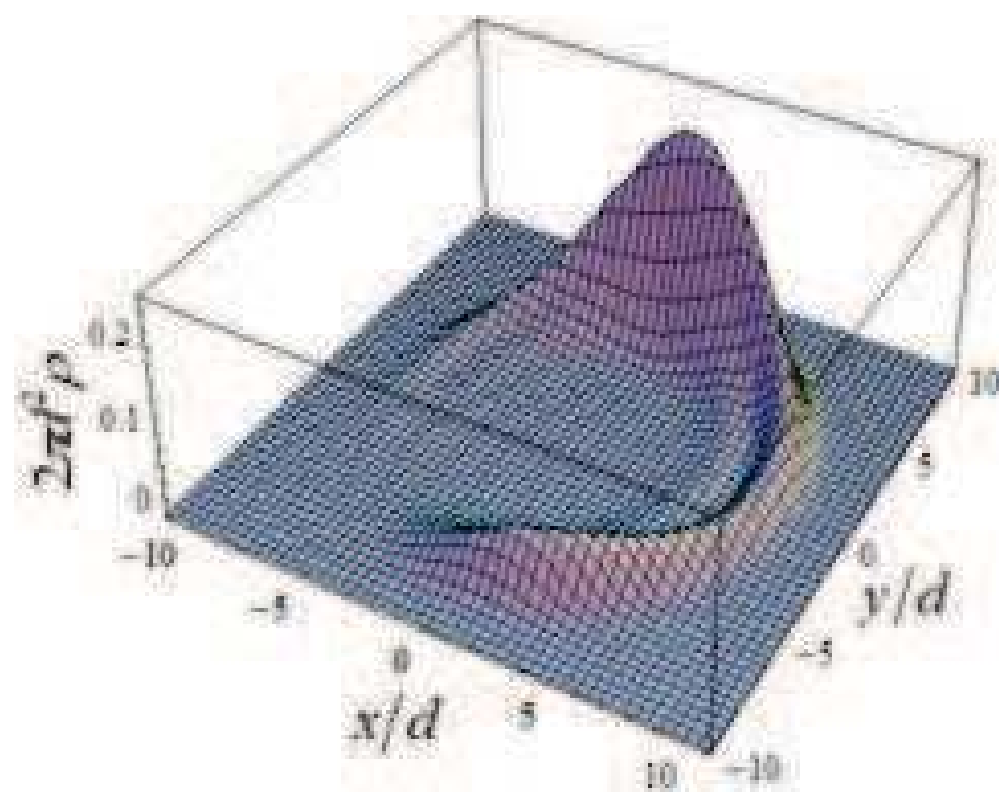

Fig. 7. (Color online). The electron probability density $\rho(\vec{r}, t)=\left|\psi_{1}\right|^{2}+\left|\psi_{2}\right|^{2}$ for initial wave packet, Eq.(8a), (8b) with $c_{1}=1$ and $c_{2}=\mathrm{e}^{i \pi / 4}$ for $a=k_{0} d=1.2$ at the time $t=7$ (in the units of $d / u)$.

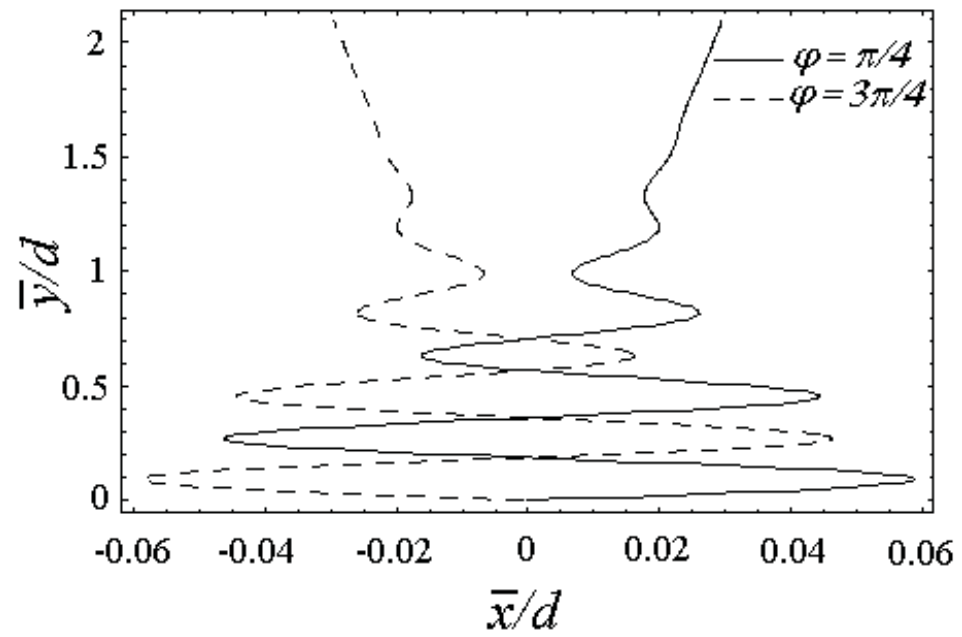

Fig. 8. The trajectories of the center of electron wave packet described by Eq.(30) for two initial phases $\varphi=\pi / 4$ and $3 \pi / 4$. The parameter $a=6$.

In particular, as it follows from Eq.(33) for $a \ll 1$, the direction of the motion of wave packet center at large time coincides with the initial one, Eq(32). In other limiting case $a \gg 1$ (and for not too small $\varphi$ ) asymptotic direction of the average velocity is along $0 Y$ axis, i.e. along the 
average momentum of wave packet $p_{y}=\hbar k_{0}$. Thus, by changing the initial phase $\varphi$, one can govern the packet motion and consequently the direction of dc current. To illustrate this, we plot in Fig. 8 the packet center trajectories for two initial phases: $\varphi=\pi / 4$ and $3 \pi / 4$. Note that the packet motion with the constant velocity predicted above (see Eqs.(22), (28)) should lead to the existence of the direct current in the corresponding direction.

To check our formalism let us consider the plane wave as the starting point. In this case it is easy to obtain the expression for the average value of electron velocity $\overline{\vec{v}}(t)$. Really, in the Heisenberg picture the kinetic velocity is (in the dimensional variables)

$$
\hat{\vec{v}}(t)=\frac{1}{i \hbar}[\vec{r}, \hat{H}]=u \vec{\sigma}(t),
$$

where

$$
\frac{d \vec{\sigma}}{d t}=\frac{1}{i \hbar}[\vec{\sigma}, \hat{H}]=\frac{2 u}{\hbar}[\hat{\vec{p}} \times \vec{\sigma}] .
$$

In these equations $\vec{p}(t)=\vec{p}(0)$. Let the initial momentum $p_{\text {oy }}=\hbar k_{0}$. Then, using the solutions of Eqs.(34), (35) we find

$$
\begin{gathered}
\bar{v}_{x}(t)=u \bar{\sigma}_{z}(0) \sin \omega t+u \bar{\sigma}_{x}(0) \cos \omega t, \\
\bar{v}_{y}(t)=u \bar{\sigma}_{y}(0)
\end{gathered}
$$

where $\omega=2 u k_{0}$ and $\sigma_{i}(0)=\sigma_{i}$ - Pauli matrixes $(i=1,2,3)$. So, if in the initial state pseudospin is along $z$ direction, i.e. $\bar{\sigma}_{z}(0)=1$ (case i)) we obtain from Eq.(34a) that $\bar{v}_{x}(t)=u \sin \omega t$ which leads to

$$
\bar{x}(t)=\text { const }-\frac{u}{\omega} \cos \omega t .
$$

Returning to the original variables in Eq.(18) and setting $d=\infty$ we see that this expression coincides with Eq.(37). We get similar results also for other initial polarizations.

\section{Concluding remarks}

We have studied the quantum dynamics of charge particles represented by Gaussian wave packets in two-dimensional single layer of carbon atoms (graphene). We investigated numerically also the spatial evolution of the initial wave packet and demonstrated the effect of the packet splitting for the pseudospin polarization perpendicular to the average momentum. The analytical expressions for the average values of position operators were obtained. These expressions clearly demonstrate that the evolution of wave function is accompanied by the Zitterbewegung and strongly depends on the initial pseudospin polarization. In particular, if the initial pseudospin polarization coincides with initial average momentum, the packet center moves and exhibits the $\mathrm{ZB}$ along the same direction. In this case the second term in Eq.(28) describing the longitudinal oscillations (the "longitudinal" ZB) is essentially smaller than the first one connected with the motion with constant velocity. As for other systems with two-band structure (Demikhovskii et al.,2008; Rusin \& Zawadzki,2007; Thaller,2004), the ZB in monolayer graphene has a transient character.

It was also predicted that apart from the packet center exhibits the trembling motion it can move with constant velocity (for example, for the pseudospin polarization along $x$ and $y$ axis). The direction of this velocity depends on not only the orientation of average momentum $\vec{p}_{0}$, 
but also on the module of the components $\psi_{1}(\vec{r}, 0), \psi_{2}(\vec{r}, 0)$ and the differences of their phases (see Eqs.(30),(33)).

All above calculations have been done for the $\vec{K}$ point of the Brillouin zone in graphene. Similar results can be found for initial wave packet with wave vector $\vec{k}$ in the valley centered in inequivalent point $\vec{K}^{\prime}$. The Dirac Hamiltonian around $\vec{K}^{\prime}$ point can be written as

$$
H_{K^{\prime}}=u\left(\begin{array}{cc}
0 & -\hat{p}_{x}-i \hat{p}_{y} \\
-\hat{p}_{x}+i \hat{p}_{y} & 0
\end{array}\right)
$$

This expression can be obtained from Hamiltronian around $\vec{K}$ point given by Eq.(1) by replacement $\hat{p}_{x} \rightarrow-\hat{p}_{x}$. Other representation of Eq.(38) are common in the literature as well. In this connection see (Bena \& Montambaux,2009; Gusynin et al.,2007). Thus values $\bar{x}(t)$ for the wave packet of different polarizations (and corresponding components of velocity) change sign while $\bar{y}(t)$ remain unchanged (see also(Rusin \& Zawadzki,2008)).

In conclusion we would like to stress that the packet motion with the constant velocity (see Eqs.(22), (28)) leads to the appearance of the dc current. For the experimental detection of this current one needs sensitive current meters. Experimental observation of trembling motion is currently a more difficult task since it is necessary to use femtosecond techniques. (Zawadzki,2006; Rusin \& Zawadzki,2007) If new methods of formation of wave packets with different pseudospin polarizations will be elaborated then their trajectories and spatial separations can be observed probably with the help of devices with quantum point contacts and gates (see for example (Castro et al.,2009)). The intresting experimental method allows an observation of ZB of electron in graphene exited by femtosecond laser pulse in the presence of magnetic field was proposed in Ref. (Rusin \& Zawadzki,2009).

\section{Appendix}

Besides the rapid oscillations $(\mathrm{ZB})$ the electron wave packet in graphene (as for other two-band systems) can drift with constant velocity $\overline{\vec{V}}_{0}$ although its average momentum is zero. The existence of constant component in the wave packet center velocity depends on the form and symmetry of the initial wave function.

Let now find the drift velocity for the arbitrary initial state, Eq.(8a). At $t>0$ the total wave function in the momentum space can be decomposed into positive- $\left(\Psi_{+}(\vec{p}, t)\right)$ and negativeenergy $\left(\Psi_{-}(\vec{p}, t)\right)$ components.

$$
\Psi(\vec{p}, t)=\Psi_{+}(\vec{p}, t)+\Psi_{-}(\vec{p}, t)=a_{+} U_{+}(\vec{p}) \mathrm{e}^{-i E_{+} t / \hbar}+l+a_{-} U_{-}(\vec{p}) \mathrm{e}^{-i E_{-} t / \hbar},
$$

Using the expression (3) for the eigenspinors $U_{ \pm}(\vec{p})$ we find the coefficients $a_{ \pm}$

$$
a_{ \pm}=\frac{F(\vec{p})}{\sqrt{2\left(\left|c_{1}\right|^{2}+\left|c_{2}\right|^{2}\right)}}\left(c_{1} \pm \frac{\left(p_{x}-i p_{y}\right)}{p} c_{2}\right)
$$

where $F(\vec{p})$ is to be determined from the Fourier expansion of coordinate wave function $f(\vec{r})$. Obviously the time-independent part $V_{\mu 0}$ of the density of velocity in the momentum space is defined as

$$
V_{\mu 0}(\vec{p})=\Psi_{+}^{+}(\vec{p}, t) \hat{V}_{\mu} \Psi_{+}(\vec{p}, t)+\Psi_{-}^{+}(\vec{p}, t) \hat{V}_{\mu} \Psi_{-}(\vec{p}, t),
$$

where $\hat{V}_{\mu}=u \sigma_{\mu}, \mu=1,2$ is velocity operator. The preceding expressions (A.1), (A.2), (A.3) immediately lead to the constant velocity of the wave packet center given in Eqs.(24), (25). 


\section{Acknowledgments}

This work was supported by the Program of the Russian Ministry of Education and Science "Development of scientific potential of High education" (Project No. 2.1.1.2686) and Grant of Russian Foundation for Basic Research (No. 09-02-01241-a), and by the President of RF Grant for Young Researchers MK-1652.2009.2.

\section{References}

[Ferrari \& Russo,1990] Ferrari, L. \& Russo, G. (1990). Nonrelativistic Zitterbewegung in two-band systems.Phys Rev. B, Vol. 42, October 1990, 7454-7461.

[Shmueli et al.,1995] Shmueli, N.; Yacoby, A. \& Imry, Y. (1995). Quantum Oscillatory Phenomena in a $p-n$ Junction of Quantum Wells. Europhys. Lett., Vol. 29, No. 9, March 1995, 711-716.

[Schliemann et al.,2005] Schliemann, J.; Loss, D. \& Westervelt, R., M. (2005). Zitterbewegung of Electronic Wave Packets in III-V Zinc-Blende Semiconductor Quantum Wells. Phys. Rev. Lett., Vol. 94, May 2005, 206801.

[Schliemann et al.,2006] Schliemann, J.; Loss, D. \& Westervelt, R., M. (2006). Zitterbewegung of electrons and holes in III-V semiconductor quantum wells. Phys. Rev. B, Vol. 73, February 2006, 085323.

[Schliemann,2008] Schliemann, J. (2005). Cyclotron motion and magnetic focusing in semiconductor quantum wells with spin-orbit coupling. Phys. Rev. B, Vol. 77, March 2008, 125303.

[Zawadzki,2005] Zawadzki, W. (2005). Zitterbewegung and its effects on electrons in semiconductors. Phys. Rev. B, Vol. 72, August 2005, 085217.

[Zawadzki,2006] Zawadzki, W. (2006). One-dimensional semirelativity for electrons in carbon nanotubes. Phys. Rev. B, Vol. 74, November 2006, 205439.

[Katsnelson,2006] Katsnelson, M., I. (2006). Zitterbewegung, chirality, and minimal conductivity in graphene. Europ. Phys. J. B, Vol. 51, No. 2, May 2006, 157-160.

[Cserti \& David,2006] Cserti, J. \& David, G. (2006). Unified description of Zitterbewegung for spintronic, graphene, and superconducting systems. Phys. Rev. B, Vol. 74, November 2006, 172305.

[Winkler et al.,2007] Winkler, R.; Zulicke, U. \& Bolter, J. (2007). Oscillatory multiband dynamics of free particles: The ubiquity of Zitterbewegung effects. Phys. Rev. B, Vol. 75, May 2007, 205314.

[Trauzettel et al.,2007] Trauzettel, B.; Blanter, Y., M. \& Morpurgo, A., F. (2007). Photon-assisted electron transport in graphene: Scattering theory analysis. Phys. Rev. B, Vol. 75, January 2007, 035305.

[Rusin \& Zawadzki,2007] Rusin, T., M. \& Zawadzki, W. (2007). Zitterbewegung of nearly-free and tightly-bound electrons in semiconductors. J. Phys. Cond. Matter, Vol. 19, No.13, March 2007, 136219.

[Rusin \& Zawadzki,2007] Rusin, T., M. \& Zawadzki, W. (2007). Transient Zitterbewegung of charge carriers in mono- and bilayer graphene, and carbon nanotubes. Phys. Rev. B, Vol. 76, November 2007, 195439.

[Rusin \& Zawadzki,2008] Rusin, T., M. \& Zawadzki, W. (2008). Zitterbewegung of electrons in graphene in a magnetic field. Phys. Rev. B, Vol. 78, September 2008, 125419. 
[Demikhovskii et al.,2008] Demikhovskii, V.,Ya.; Maksimova, G., M. \& Frolova, E., V. (2008). Wave packet dynamics in a two-dimensional electron gas with spin orbit coupling: Splitting and Zitterbewegung. Phys. Rev. B, Vol. 78, September 2008, 115401.

[Schrödinger,1930] Schrödinger, E. (1930). Über die kräftefreie Bewegung in der relativistischen Quantenmechanik. Sitzungsber. Peuss. Akad. Wiss. Phys. Math. Kl., Vol. 24, 1930, 418-428.

[Barut \& Bracken,1981] Barut, A., O. \& Bracken, A., J. (1981). Zitterbewegung and the internal geometry of the electron.Phys. Rev. D, Vol. 23, May 1981, 2454-2463.

[Lock,1979] Lock, J., A. (1979). The Zitterbewegung of a free localized Dirac particle. Am. J. Phys., Vol. 47, September 1979, 797-802.

[Thaller,2004] Thaller, B. (2004). Visualizing the kinematics of relativistic wave packets. arXiv: quant-ph/0409079v1, September 2004, 1-7.

[Braun et al.,1999] Braun, J., W.; Su, Q. \& Grobe, R. (1999). Numerical approach to solve the time-dependent Dirac equation. Phys. Rev. A, Vol. 59, January 1999, 604-612.

[Demikhovskii et al.,2010] Demikhovskii,V.,Ya.; Maksimova,G., M.; Perov, A., A. \& Frolova,E., V. (2010). Space-time evolution of Dirac wave packets. Phys.Rev A, Vol.82, November 2010, 052115.

[Gerritsma et al.,2010] Gerritsma, R.; Kirchmair, G.; Zähringer, F.; Solano, E.; Blatt, R. \& Roos, C., F. (2010). Quantum simulation of the Dirac equation. Nature, Vol. 463, January 2010, 68-71.

[Lamata et al.,2007] Lamata, L.; Leon, J.; Schätz, T. \& Solana, E. (2007). Dirac Equation and Quantum Relativistic Effects in a Single Trapped Ion. Phys. Rev. Lett., Vol. 98, June 2007, 253005.

[Maksimova et al.,2008] Maksimova, G., M.; Demikhovskii, V., Ya. \& Frolova, E., V. (2008). Wave packet dynamics in a monolayer graphene. Phys. Rev. B, Vol. 78, December 2008, 235321.

[Wallace,1947] Wallace, P., R. (1947). The Band Theory of Graphite. Phys. Rev., Vol. 71, May 1947, 622-634.

[Slonczewski \& Weiss,1958] Slonczewski, J., C. \& Weiss, P., R. (1958). Band Structure of Graphite. Phys. Rev., Vol. 109, January 1958, 272-279.

[Novoselov et al.,2005] Novoselov, K., S.; Geim, A., K.; Morozov, S., V.; Jiang, D., Katsnelson, M., I.; Grigorieva, I., V.; Dubonos, S., V. \& Firsov, A., A. (2005). Two-dimensional gas of massless Dirac fermions in graphene. Nature, Vol. 438, November 2005, 197-200.

[Novoselov et al.,2006] Novoselov, K., S.; Cann, E., Mc.; Morozov, S., V.; Falko, V., I.; Katsnelson, M., I.; Zeitler, U., Yiang, D.; Schedin, F. \& Geim, A., K. (2006). Unconventional quantum Hall effect and Berry's phase of $2 \mathrm{n}$ in bilayer graphene. Nature Phys., Vol. 2, February 2006, 177-180.

[Zhang et al.,2005] Zhang, Y.; Tan, Y., W.; Stormer, H., L. \& Kim, P. (2005). Experimental observation of the quantum Hall effect and Berry's phase in graphene. Nature, Vol. 438, November 2005, 201-204.

[Rusin \& Zawadzki,2008] Rusin, T., M. \& Zawadzki, W. (2008). Zitterbewegung of electrons in graphene in a magnetic field. Phys. Rev. B, Vol. 78, September 2008, 125419.

[Bena \& Montambaux,2009] Bena, C. \& Montambaux, G. (2009). Remarks on the tight-binding model of graphene. New J. Phys., Vol. 11, September 2009, 095003. 
[Gusynin et al.,2007] Gusynin, V., P.; Sharapov, S., G. \& Carbotte, J., P. (2007). AC conductivity of graphene: from tight-binding model to 2+1-dimensional quantum electrodynamics. Int.J.Mod.Phys.B, Vol. 21, No. 27, October 2007, 4611-4658.

[Castro et al.,2009] Castro Neto, A., H.; Guinea, F., Peres, N., M.; Novoselov, K., S. \& Geim, A., K. (2009). The electronic properties of graphene.Rev. Mod. Phys., Vol. 81, January 2009, 109-162.

[Rusin \& Zawadzki,2009] Rusin, T., M. \& Zawadzki, W. (2009). Theory of electron Zitterbewegung in graphene probed by femtosecond laser pulses. Phys. Rev. B, Vol. 80, July 2009, 045416. 


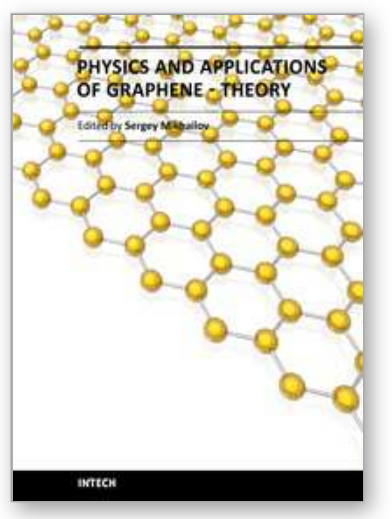

\section{Physics and Applications of Graphene - Theory}

Edited by Dr. Sergey Mikhailov

ISBN 978-953-307-152-7

Hard cover, 534 pages

Publisher InTech

Published online 22, March, 2011

Published in print edition March, 2011

The Stone Age, the Bronze Age, the Iron Age... Every global epoch in the history of the mankind is characterized by materials used in it. In 2004 a new era in material science was opened: the era of graphene or, more generally, of two-dimensional materials. Graphene is the strongest and the most stretchable known material, it has the record thermal conductivity and the very high mobility of charge carriers. It demonstrates many interesting fundamental physical effects and promises a lot of applications, among which are conductive ink, terahertz transistors, ultrafast photodetectors and bendable touch screens. In 2010 Andre Geim and Konstantin Novoselov were awarded the Nobel Prize in Physics "for groundbreaking experiments regarding the two-dimensional material graphene". The two volumes Physics and Applications of Graphene - Experiments and Physics and Applications of Graphene - Theory contain a collection of research articles reporting on different aspects of experimental and theoretical studies of this new material.

\section{How to reference}

In order to correctly reference this scholarly work, feel free to copy and paste the following:

G. M. Maksimova, V. Ya. Demikhovskii, and E. V. Frolova (2011). Wave Packet Dynamics in a Monolayer Graphene, Physics and Applications of Graphene - Theory, Dr. Sergey Mikhailov (Ed.), ISBN: 978-953-307152-7, InTech, Available from: http://www.intechopen.com/books/physics-and-applications-of-graphenetheory/wave-packet-dynamics-in-a-monolayer-graphene

\section{INTECH}

open science | open minds

\section{InTech Europe}

University Campus STeP Ri

Slavka Krautzeka 83/A

51000 Rijeka, Croatia

Phone: +385 (51) 770447

Fax: +385 (51) 686166

www.intechopen.com

\section{InTech China}

Unit 405, Office Block, Hotel Equatorial Shanghai

No.65, Yan An Road (West), Shanghai, 200040, China 中国上海市延安西路65号上海国际贵都大饭店办公楼 405 单元

Phone: +86-21-62489820

Fax: $+86-21-62489821$ 
(C) 2011 The Author(s). Licensee IntechOpen. This chapter is distributed under the terms of the Creative Commons Attribution-NonCommercialShareAlike-3.0 License, which permits use, distribution and reproduction for non-commercial purposes, provided the original is properly cited and derivative works building on this content are distributed under the same license. 